\title{
Low-dimensional fluctuations and pseudogap in Gaudin-Yang Fermi gases
}

\author{
Hiroyuki Tajima $\odot,{ }^{1}$ Shoichiro Tsutsui, ${ }^{2}$ and Takahiro M. Doi ${ }^{3}$ \\ ${ }^{1}$ Department of Mathematics and Physics, Kochi University, Kochi 780-8520, Japan \\ ${ }^{2}$ Quantum Hadron Physics Laboratory, RIKEN Nishina Center, Wako, Saitama, 351-0198, Japan \\ ${ }^{3}$ Research Center for Nuclear Physics (RCNP), Osaka University, Osaka 567-0047, Japan
}

(Received 31 May 2020; revised 3 August 2020; accepted 28 August 2020; published 18 September 2020)

\begin{abstract}
The pseudogap is a ubiquitous phenomenon in strongly correlated systems, such as high- $T_{\mathrm{c}}$ superconductors, ultracold atoms, and nuclear physics. Whereas pairing fluctuations inducing the pseudogap are known to be enhanced in low-dimensional systems, such effects have not been explored well in one of the most fundamental one-dimensional models, that is, Gaudin-Yang model. In this paper, we show how the pseudogap effect emerges in the single-particle excitation in this system using a diagrammatic approach. Fermionic single-particle spectra exhibit a unique crossover from the double-particle dispersion to the pseudogap state with increasing the attractive interaction and the number density at finite temperature. Surprisingly, our results of thermodynamic quantities in unpolarized and polarized gases show an excellent agreement with the recent quantum Monte Carlo and complex Langevin results, indicating the validity of our approach even in the region where the pseudogap appears.
\end{abstract}

DOI: 10.1103/PhysRevResearch.2.033441

\section{INTRODUCTION}

A pseudogap phenomenon, which is the suppression of the density of states (DOS) around a Fermi level, has been a central issue in strongly correlated quantum many-body systems such as high- $T_{\mathrm{c}}$ superconductors [1-5], ultracold atoms [6-14], and nuclear and quark matter [15-20]. Although the origin of the pseudogap strongly depends on the properties of each system, it is believed that the pseudogap is induced by fluctuation effects dominating nontrivial characters of the systems. Recently, an ultracold atomic gas provides us an ideal platform to study pseudogap physics and associated fluctuation effects in a systematic manner [21-33], thanks to the realization of the Bardeen-Cooper-Schrieffer (BCS) to Bose-Einstein-condensation (BEC) crossover [34-40].

Furthermore, the low dimensionality tends to induce strong fluctuations [41,42]. It is a key point also for properties of carbon nanotubes [43,44], organic conductors [45-47], as well as nuclear pasta in neutron star crusts [48,49]. In ultracold atom physics, two-dimensional pseudogap effects have attracted much attention [50-60] because these many-body effects are expected to be more visible than three-dimensional (3D) systems. Along this direction, the pseudogap in a onedimensional (1D) cold atomic system would be a fascinating topic.

Although an attractively interacting two-component Fermi gas in 1D, namely, Gaudin-Yang model is known as a solvable model based on the thermodynamic Bethe ansatz (TBA) [61], it does not mean that physical quantities we are interested in

Published by the American Physical Society under the terms of the Creative Commons Attribution 4.0 International license. Further distribution of this work must maintain attribution to the author(s) and the published article's title, journal citation, and DOI. can easily be obtained in an exact way. Low-energy effective field-theory descriptions, such as Tomonaga-Luttinger liquid [62] have also been employed frequently in 1D. Although such approaches also give exact results at zero temperature, it is not the case at finite temperature where the Fermi step is softened. Precise results of this 1D fermionic system at finite temperature were obtained by TBA [63-65] and a recent state-of-the-art work of quantum Monte Carlo (QMC) simulation performed by Hoffman et al. [66]. Afterwards, various thermal properties of this system have been investigated within a lattice simulation [67-70]. However, no one shows how the pseudogap phenomena occur in this famous 1D model. Moreover, the possibility of an inhomogeneous pairing state called Fulde-Ferrel-Larkin-Ovchinikov- (FFLO)-like state [71,72] has also been extensively investigated in a spinimbalanced 1D system [73-79] since the 1D FFLO-like state is expected to be robust against fluctuations. To see this, a quantitative analysis of fluctuation effects are really desired. It involves interdisciplinary interests from other fields. In the context of quantum chromodynamics (QCD), the FFLO-like state of quark-antiquark pairs called chiral spiral is anticipated at finite density [80-82].

In this paper, we elucidate pairing fluctuation effects in 1D Gaudin-Yang Fermi gas at finite temperature within the diagrammatic approach, which has successfully been applied to higher-dimensional systems $[6,9,11,12,14]$. Our numerical results of the number density show an excellent agreement with the recent QMC results [66]. In the polarized case, we show that our result also well reproduces a complex Langevin (CL) simulation [83], which is a promising candidate for overcoming a sign problem in an imbalanced Fermi gas [84]. Furthermore, we show that the single-particle excitation spectra exhibit the pseudogapped structure due to pairing fluctuations in the region where the validity is guaranteed by the comparison with QMC results for the thermodynamic quantity. 


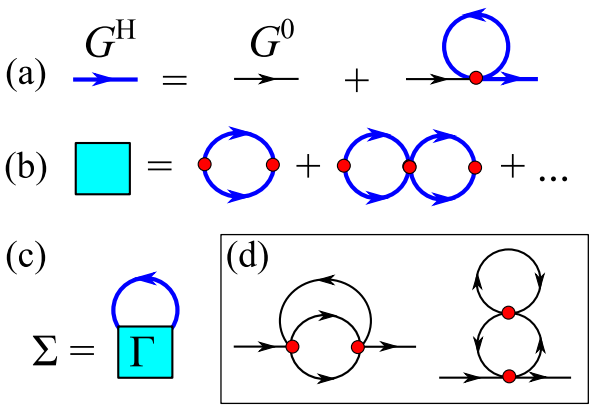

FIG. 1. Feynman diagrams for (a) the Hartree-Green's function $G^{\mathrm{H}}$ (solid), (b) four-point vertex $\Gamma$, (c) the self-energy $\Sigma$, and (d) the second-order connected diagrams taken into account in our approach. The thin line denotes the bare Green's function $G^{0}$.

This paper is organized as follows. In Sec. II, we explain our formalism of a diagrammatic strong-coupling approach. In Sec. III, we show our results of number densities as functions of chemical potentials and temperatures and compare them with QMC results. We discuss how the pseudogap emerges in single-particle excitation properties at finite temperature. Finally, we compare our results of the polarization at finite chemical potential differences with the CL results. In Sec. IV, we make a summary of this paper. Throughout the paper, we set $\hbar=k_{\mathrm{B}}=1$, and the system size $L$ is taken to be unity.

\section{FORMALISM}

We start from the attractive Gaudin-Yang model described by the Hamiltonian,

$$
\begin{aligned}
H= & \sum_{p, \sigma} \xi_{p, \sigma} c_{p, \sigma}^{\dagger} c_{p, \sigma}+g \sum_{k, k^{\prime}, q} c_{k+q / 2, \uparrow}^{\dagger} c_{-k+q / 2, \downarrow}^{\dagger} \\
& \times c_{-k^{\prime}+q / 2, \downarrow} c_{k^{\prime}+q / 2, \uparrow},
\end{aligned}
$$

where $\xi_{p, \sigma}=p^{2} /\left(2 m_{\sigma}\right)-\mu_{\sigma}$ is the kinetic energy of a fermion with momentum $p$, spin $\sigma=\uparrow, \downarrow$, and mass $m_{\sigma}$ measured from the chemical potential $\mu_{\sigma}$. For simplicity, we consider the mass-balanced case $\left(m \equiv m_{\uparrow}=m_{\downarrow}\right)$. $\mu_{\sigma}$ is parametrized by the averaged one $\mu=\left(\mu_{\uparrow}+\mu_{\downarrow}\right) / 2$ and a fictitious magnetic-field $h=\left(\mu_{\uparrow}-\mu_{\downarrow}\right) / 2 . c_{p, \sigma}$ and $c_{p, \sigma}^{\dagger}$ are fermionic annihilation/creation operators, respectively. The coupling constant $g$ is related to a 1D scattering length $a$ as $g=-\frac{2}{m a}$. Following Ref. [66], we measure the interaction strength through the dimensionless parameter $\lambda^{2}=m g^{2} / T$. Since the two-body bound state with the binding energy $E_{\mathrm{b}}=$ $1 /\left(m a^{2}\right)$ always exists in an attractive $1 \mathrm{D}$ system, $\lambda^{2}=4 E_{\mathrm{b}} / T$ characterizes the ratio between $E_{\mathrm{b}}$ and $T$.

The important note is that, whereas in two-dimensional and 3D systems, the contact-type interaction becomes zero such that a finite scattering length is reproduced [85], it is not the case in this 1D system. Therefore, the lowest-order diagram, that is, Hartree self-energy $\Sigma_{\sigma}^{\mathrm{H}}$ is nonzero, in contrast to higher-dimensional systems. To retain it as shown in Fig. 1(a), we introduce the single-particle Green's function $G_{\sigma}^{\mathrm{H}}\left(p, i \omega_{n}\right)$ with the Hartree shift $\Sigma_{\sigma}^{\mathrm{H}}$ given by

$$
G_{\sigma}^{\mathrm{H}}\left(p, i \omega_{n}\right)=G_{\sigma}^{0}\left(p, i \omega_{n}\right)\left[1+\Sigma_{\sigma}^{\mathrm{H}} G_{\sigma}^{\mathrm{H}}\left(p, i \omega_{n}\right)\right],
$$

where $\omega_{n}=(2 n+1) \pi T$ is the fermion Matsubara frequency and $-\sigma$ represents an opposite spin for $\sigma . G_{\sigma}^{0}\left(p, i \omega_{n}\right)=$ $\left[i \omega_{n}-\xi_{p, \sigma}\right]^{-1}$ is a bare Green's function. $\Sigma_{\sigma}^{\mathrm{H}}=g n_{-\sigma}^{\mathrm{H}}$ is self-consistently determined by solving the Hartree density equation,

$$
n_{\sigma}^{\mathrm{H}}=T \sum_{p, i \omega_{n}} G_{\sigma}^{\mathrm{H}}\left(p, i \omega_{n}\right)=\sum_{p} f\left(\xi_{p, \sigma}^{\mathrm{H}}\right),
$$

where $\xi_{p, \sigma}^{\mathrm{H}}=\xi_{p, \sigma}+g n_{-\sigma}^{\mathrm{H}} \cdot f(\xi)=\left(e^{\xi / T}+1\right)^{-1}$ is the FermiDirac distribution function. A similar approximation has been employed in nuclear physics with finite-range interactions [86-89]. On the basis of $G_{\sigma}^{\mathrm{H}}\left(p, i \omega_{n}\right)$, we incorporate pairing fluctuation effects described by the four-point vertex $\Gamma$ diagrammatically shown in Fig. 1(b), which reads

$$
\Gamma\left(q, i v_{\ell}\right)=-\frac{g^{2} \Pi\left(q, i v_{\ell}\right)}{1+g \Pi\left(q, i v_{\ell}\right)},
$$

where

$$
\begin{aligned}
\Pi\left(q, i v_{\ell}\right)= & T \sum_{k, i \omega_{n}} G_{\uparrow}^{\mathrm{H}}\left(k+\frac{q}{2}, i v_{\ell}+i \omega_{n}\right) \\
& \times G_{\downarrow}^{\mathrm{H}}\left(-k+\frac{q}{2},-i \omega_{n}\right)
\end{aligned}
$$

is the lowest-order particle-particle bubble with the boson Matsubara frequency $\nu_{\ell}=2 \ell \pi T$. The self-energy $\Sigma_{\sigma}$ for the fluctuation correction is given by

$$
\Sigma_{\sigma}\left(p, i \omega_{n}\right)=T \sum_{q, i v_{\ell}} \Gamma\left(q, i v_{\ell}\right) G_{-\sigma}^{\mathrm{H}}\left(q-p, i v_{\ell}-i \omega_{n}\right)
$$

We note that this approximation is equivalent to the so-called $T$-matrix approach, except for the self-consistent treatment of the Hartree shift. The $T$-matrix approach successfully reproduces the exact results obtained by TBA for 1D Fermi polaronic excitations realized in spin-polarized limit [90]. In our approach, by taking $G^{\mathrm{H}}\left(p, i \omega_{n}\right)$ with density mean-field $\Sigma_{\sigma}^{\mathrm{H}}$ as a building block of fluctuation corrections, at least, we take all possible connected diagrams into account up to the second-order shown in Fig. 1(d). Using the dressed Green's function,

$$
G_{\sigma}\left(p, i \omega_{n}\right)=\frac{G_{\sigma}^{\mathrm{H}}\left(p, i \omega_{n}\right)}{1-\Sigma_{\sigma}\left(p, i \omega_{n}\right) G_{\sigma}^{\mathrm{H}}\left(p, i \omega_{n}\right)},
$$

we obtain the number density $n_{\sigma}$ for given $T$ and $\mu_{\sigma}$ as

$$
n_{\sigma}=T \sum_{p, i \omega_{n}} G_{\sigma}\left(p, i \omega_{n}\right) \text {. }
$$

Moreover, we can obtain the single-particle spectral function,

$$
A_{\sigma}(p, \omega)=-\frac{1}{\pi} \operatorname{Im} G_{\sigma}\left(p, i \omega_{n} \rightarrow \omega+i \delta\right),
$$

and the DOS,

$$
\rho_{\sigma}(\omega)=\sum_{p} A_{\sigma}(p, \omega),
$$

from $G_{\sigma}\left(p, i \omega_{n}\right)$. In what follows, we suppress $\sigma$ in these quantities as $n, A(p, \omega)$, and $\rho(\omega)$ unless otherwise specified. 


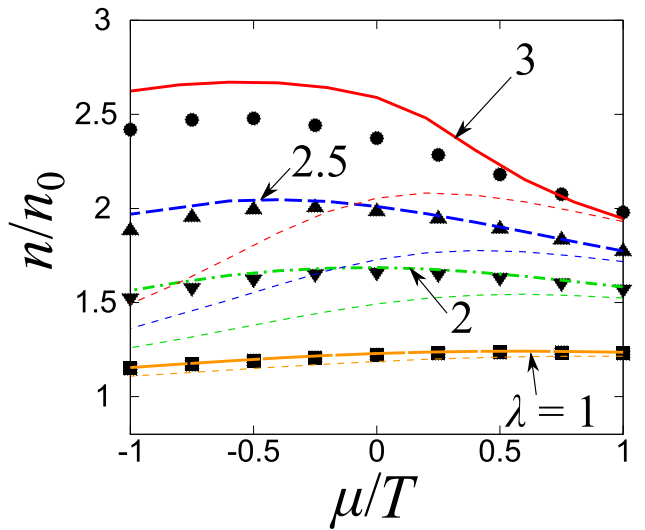

FIG. 2. Calculated number density $n / n_{0}$ as function of $\mu / T$ in an unpolarized system, where $n_{0}$ is the noninteracting counterpart. The thick and thin curves show the numerical results of our diagrammatic approach and the Hartree-Fock calculation given by Eq. (3), respectively. The interaction parameter $\lambda$ is given by $1,2,2.5$, and 3 from the bottom. The black symbols represent 1D QMC results [66] of $\lambda=1$ (square), 2 (inverted triangle), 2.5 (triangle), and 3 (circle).

\section{RESULTS}

Figure 2 shows the calculated number density $n / n_{0}$ as a function of $\mu / T$ in an unpolarized gas, where $n_{0}$ is the noninteracting counterpart. Our results given by thick curves show an excellent agreement with 1D QMC results from Ref. [66]. For comparison, we also plot the Hartree-Fock results (thin curves) given by $n^{\mathrm{H}}$ in Eq. (3). Although all results coincides with each other in the weak-coupling regime, such as $\lambda=1$, the Hartree-Fock result deviates from the others due to the lack of fluctuation effects. Our main results well reproduce the QMC results even in the strong-coupling regime $(\lambda \geqslant 2)$ where $E_{\mathrm{b}}=\lambda^{2} T / 4 \geqslant T$. Although the result at $\lambda=3$ is close to the applicable limit of our approach as we will mention later, still it shows a semiquantitative agreement with QMC. In this way, we can check the validity of our approach in these parameter regimes. We note that these results are also consistent with the TBA [91].

Figures 3(a1), 3(b1), and 3(c1) show the calculated DOS $\rho(\omega)$ at $\lambda=2,2.5$, and 3 . One can see the dip structure around $\omega=0$ (corresponding to the Fermi level) with the double peaks in the wide parameter region. Although the higherenergy peak locates at $\omega=-\mu$ in the low-density regime $(\mu / T \lesssim 0)$, the energy of lower one is approximately given by $\omega=-E_{\mathrm{b}} / 2$. This indicates the existence of two-body bound molecules. With increasing the density (in other words, $\mu / T$ or $\lambda$ ), one can find a pronounced gap structure even in the high-density regime $(\mu / T \gtrsim 0)$. It is expected to originate from many-body effects, namely, pairing fluctuations associated with the Cooper instability.

Although the dip structures in Fig. 3 are similar to the pseudogap, we have to carefully distinguish the fluctuationinduced pseudogap and the double peak due to the two-body bound state. For this purpose, $A(p, \omega)$ is useful. At an intermediate coupling $(\lambda=2)$ in Fig. 3(a2), $A(p, \omega)$ is largely broadened around $\omega=0$. Although the obtained spectra are somewhat similar to those in the Luther-Emery model at $T=0[92,93]$, the broadening and renormalization of the
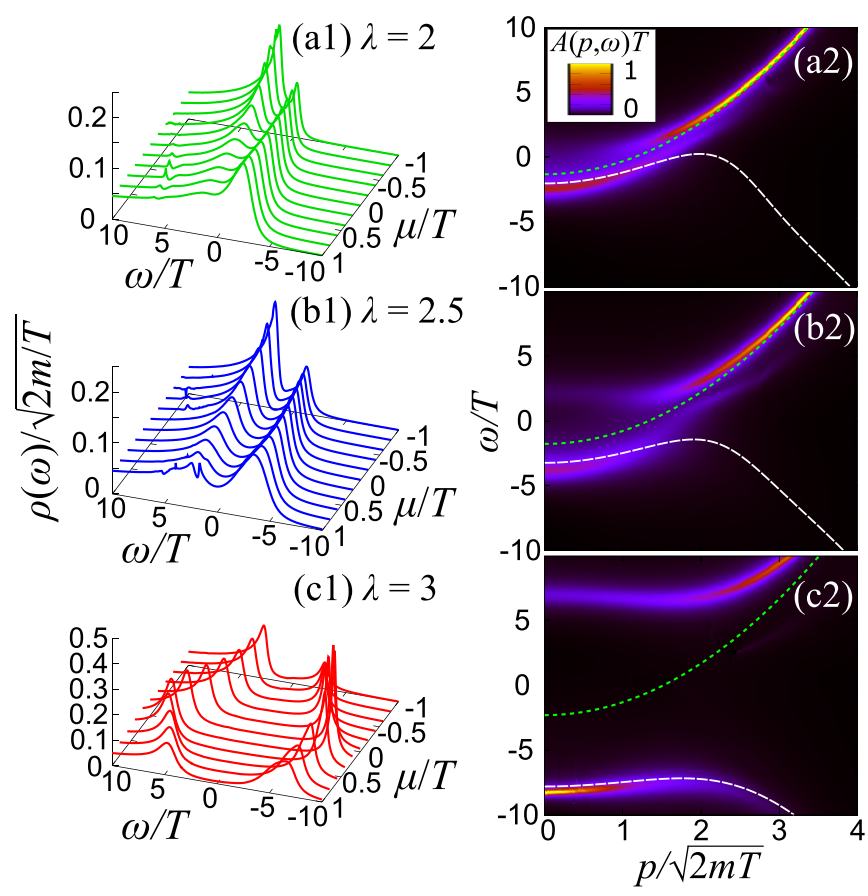

FIG. 3. Calculated DOS $\rho(\omega)$ with $h=0$ at (a1) $\lambda=2$, (b1) 2.5 , and (c1) 3. Panels (a2), (b2), and (c2) show the corresponding singleparticle spectral functions $A(p, \omega)$ at $\mu / T=0.4$. In the right panels, the dashed and dotted curves represent $\xi_{p, \sigma}^{\text {eff }}$ obtained from Eq. (12) with $G$ and $G^{\mathrm{H}}$, respectively.

dispersion through the self-energy corrections are significant even in the relatively high-energy region $(|\omega| \gtrsim T)$. The double-particle (two quadratic) dispersions in Fig. 3(a2) can be qualitatively understood from the crossover between high-momentum bare atoms and dressed atoms through the self-energy at small momenta. A similar spectrum can be found in the strong-coupling BEC regime in higher dimensions $[24,55]$. We note that the high-momentum sharp peak is shifted by the Hartree correction $\Sigma^{\mathrm{H}}$. With increasing the interaction strength as shown in Fig. 3(b2), the two dispersions are separated, and a holelike contribution appears at positive energy. The overall structure gradually changes into the pseudogapped spectrum. At stronger coupling in Fig. 3(c2) since the low-energy pole in $\Gamma\left(q, i v_{\ell}\right)$ becomes close to $q=0$ and gives a strong particle-hole coupling, one can clearly see the pseudogap accompanying with particlehole branches. Intuitively, this pseudogap structure can be understood from the so-called static approximation where $\Sigma_{\sigma}^{\mathrm{pg}}\left(p, i \omega_{n}\right) \simeq-\Delta_{\mathrm{pg}}^{2} G_{-\sigma}^{\mathrm{H}}\left(-p,-i \omega_{n}\right) \quad[12,14]$. Here, $\Delta_{\mathrm{pg}}^{2}=$ $-T \sum_{q, i v_{\ell}} \Gamma\left(q, i v_{\ell}\right)$ is called the pseudogap parameter which characterizes its size in $A(p, \omega)$ as well as $\rho(\omega)$. Indeed, this approximated self-energy induce the BCS-like propagator $G_{\sigma}^{\mathrm{pg}}\left(p, i \omega_{n}\right)$ as

$$
G_{\sigma}^{\mathrm{pg}}\left(p, i \omega_{n}\right)=\frac{i \omega_{n}+\xi_{-p,-\sigma}^{\mathrm{H}}}{\left(i \omega_{n}-\xi_{p, \sigma}^{\mathrm{H}}\right)\left(i \omega_{n}+\xi_{-p,-\sigma}^{\mathrm{H}}\right)-\Delta_{\mathrm{pg}}^{2}},
$$

which leads to the BCS-like gapped DOS. These results indicate the crossover from the superposition of bare and dressed atoms to the pseudogap state with increasing the interaction at finite temperature. The pseudogapped dispersion of fermions 
yields that the elementary excitation is now replaced by the bosonic two-particle excitations [94]. We note that, in this paper, we do not specify the crossover boundary between the pseudogap regime and the bound molecular regime since its definition involves ambiguity $[11,12,14]$.

We also compare the dispersion $\xi_{p, \sigma}^{\text {eff }}$ obtained from the imaginary-time Green's function $G_{\sigma}(p, \tau)$ as

$$
\xi_{p, \sigma}^{\mathrm{eff}}=-\frac{1}{\Delta \tau} \ln \left|\frac{G_{\sigma}(p, \tau+\Delta \tau)}{G_{\sigma}(p, \tau)}\right|_{\tau \rightarrow \beta},
$$

where $\Delta \tau$ is a small number and $\beta=1 / T$ is the inverse temperature. We take $\Delta \tau=\beta / 40$, which is small enough to extract $\xi_{p, \sigma}^{\text {eff }}$. Here, $G_{\sigma}(p, \tau)$ is obtained by the Fourier transformation,

$$
G_{\sigma}(p, \tau)=T \sum_{i \omega_{n}} G_{\sigma}\left(p, i \omega_{n}\right) e^{-i \omega_{n} \tau} .
$$

We note that a large- $\tau$ limit $(\tau \rightarrow \beta)$ is required to obtain the ground-state single-particle energy. Such an extraction of the dispersion has frequently been performed to obtain hadronic spectra in lattice QCD simulations [95]. Indeed, in the single-particle case in vacuum with $G_{\sigma}(p, \tau) \propto e^{-\left(p^{2} / 2 m\right) \tau}$, one can obtain $\xi_{p, \sigma}^{\text {eff }}=p^{2} /(2 m)$ from Eq. (12). In the present case with strongly correlated media, whereas, at small momenta, $\xi_{p, \sigma}^{\text {eff }}$ well reproduces the peak in $A_{\sigma}(p, \omega)$ (see the dashed curves in the right panels in Fig. 3), it deviates from the peak at the weaker-coupling side due to the broadening of spectra as well as level couplings at high momenta. In such a high-energy regime where interaction effects are irrelevant, the dispersion obtained from $G_{\sigma}^{\mathrm{H}}(p, \tau)=\left[f\left(\xi_{p, \sigma}^{\mathrm{H}}\right)-1\right] e^{-\xi_{p, \sigma}^{\mathrm{H}} \tau}$ agrees with the spectral peak. We note that, in the presence of $\mu_{\sigma}$ and a constant shift $\Sigma_{\sigma}^{\mathrm{H}}$ without fluctuation effects, we can obtain $\xi_{p, \sigma}^{\mathrm{eff}}=\xi_{p, \sigma}^{\mathrm{H}}$ from Eq. (12) with $G_{\sigma}^{\mathrm{H}}\left(p, i \omega_{n}\right)$. These results, indeed, indicate that, although the high-momentum region can be explained by bare atomic spectra as seen in Fig. 3(a2), the low-momentum excitation involves strong pairing fluctuations due to the self-energy correction. Moreover, in the deep inside of the pseudogap regime, such as Fig. 3(c2), $\xi_{p, \sigma}^{\mathrm{eff}}$ shows good agreement with the so-called backbending curve in $A(p, \omega)$. We note that this quantity can be measured in the lattice simulation without analytic continuations. The medium corrections on $\xi_{p, \sigma}^{\text {eff }}$ in many-body systems would be useful information for the future investigation of finite-density lattice QCD simulations.

We note that our diagrammatic approach has an artificial limitation in the strong-coupling regime. Figure 4 shows the denominator of $\Gamma(q, 0)$. Whereas, in 3D systems, the superfluid transition is identified by $1+g \Pi(0,0)=0$, it should be positive due to the Mermin-Wagner-Hohenberg theorem [96,97] proving no phase transitions in uniform 1D systems. On the other hand, we encounter the zero crossing of $\Gamma(q, 0)$ around $\lambda \gtrsim 3.1$ due to the lack of higher-fluctuation corrections. However, we emphasize that our results show nontrivial spectral structures even in the region where our approach is valid and the calculated number density quantitatively agrees with the QMC results.

One may concern that, in 1D systems, the Peierls instability also occurs through the density fluctuations. In such a case, it is needed to incorporate particle-hole fluctuation

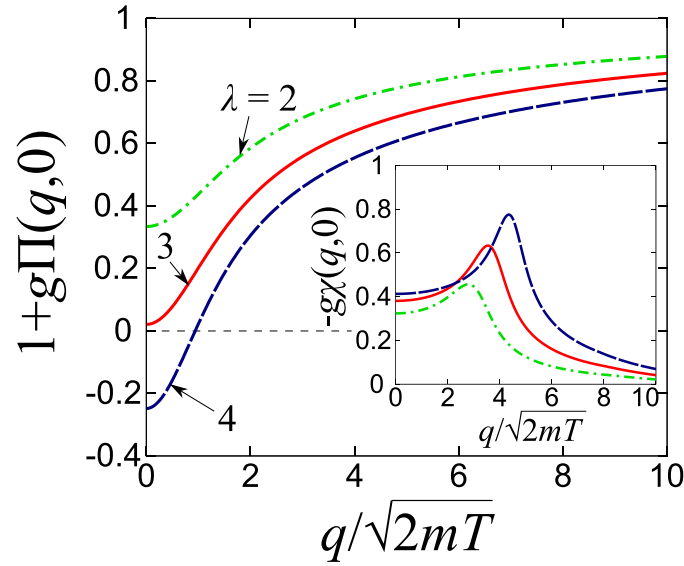

FIG. 4. The denominator of $\Gamma(q, 0)$ given by $1+g \Pi(q, 0)$ at $\mu / T=1$. The inset shows the Lindhard function $-g \chi(q, 0)$ at finite temperature.

effects by using more sophisticated approaches, such as fluctuation-exchange (FLEX) approximation [2] and parquet approximation $[98,99]$. However, this instability is suppressed by finite temperature effects. To see this, we calculate the density response function $\chi\left(q, i v_{\ell}\right)$ given by

$$
\chi\left(q, i v_{\ell}\right)=-\sum_{k} \frac{f\left(\xi_{k-q, \sigma}^{\mathrm{H}}\right)-f\left(\xi_{k, \sigma}^{\mathrm{H}}\right)}{i v_{\ell}+\xi_{k-q, \sigma}^{\mathrm{H}}-\xi_{k, \sigma}^{\mathrm{H}}} .
$$

It is nothing but the Lindhard function [100], which is known to show the logarithmic divergence with respect to $T$ at $q=2 k_{\mu} \equiv 2 \sqrt{2 m\left(\mu-\Sigma^{\mathrm{H}}\right)}$ and $v_{\ell}=0$ in 1D. One can easily find that $\chi\left(q, i v_{\ell}\right)$ disappears in the high-temperature or low-density limit where the Fermi-Dirac distribution function becomes zero $[f(\xi) \rightarrow 0]$. On the other hand, the particleparticle bubble involves a temperature-independent term as

$$
\begin{aligned}
\Pi(q, 0)= & \frac{m}{2 \sqrt{q^{2} / 4-2 m \mu}} \\
& -\sum_{k} \frac{f\left(\xi_{k+q / 2, \uparrow}^{\mathrm{H}}\right)+f\left(\xi_{-k+q / 2, \downarrow}^{\mathrm{H}}\right)}{\xi_{k+q / 2, \uparrow}^{\mathrm{H}}+\xi_{-k+q / 2, \downarrow}^{\mathrm{H}}} .
\end{aligned}
$$

In Eq. (15), the first term survives even in the limit of $f(\xi) \rightarrow 0$. In this way, with increasing the temperature (otherwise approaching the low-density limit), the particle-particle ladder resummations employed in this paper become more important than those of particle-hole bubbles for density and spin fluctuations even in 1D. The inset of Fig. 4 shows the calculated $-g \chi(q, 0)$ at $\mu / T=1$. Although $-g \chi(q, 0)$ exhibits a maximum around $q=2 k_{\mu}$, indeed, it is still finite due to the finite temperature effect. Such a softening of the anomaly in $\chi(q, 0)$ is one of the reasons why our diagrammatic approach unexpectedly well reproduces the QMC results at finite temperature. Even in the analysis based on the random-phase approximation for the density channel since the Peierls instability is identified by $1+g \chi(q, 0)=0$, the region where we explore in this paper is safely far away from this instability. 


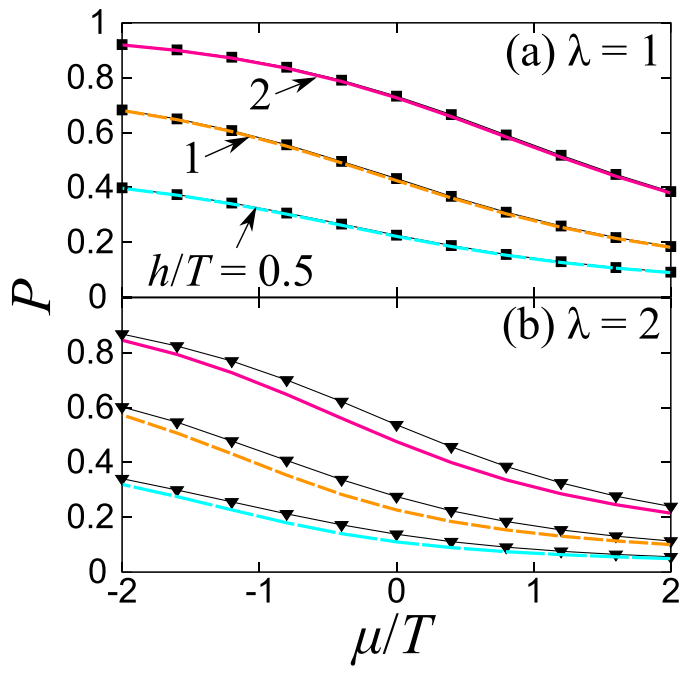

FIG. 5. The polarization equation of state $P=\left(n_{\uparrow}-n_{\downarrow}\right) /\left(n_{\uparrow}+\right.$ $n_{\downarrow}$ ) at (a) $\lambda=1$ and (b) $\lambda=2$. The calculated $P$ in our diagrammatic approach is plotted at $h / T=0.5,1$, and 2 from the bottom to the top in each figure. The filled symbols show the numerical results of the CL method [83].

We note that $\chi\left(q, i v_{\ell}\right)$ is involved in the second-order selfenergy diagram $\Sigma_{\sigma}^{2 \text { nd }}\left(p, i \omega_{n}\right)$ in our approach as

$$
\begin{aligned}
\Sigma_{\sigma}^{2 \mathrm{nd}}\left(p, i \omega_{n}\right)= & g^{2} T \sum_{q, i v_{\ell}} \chi\left(q, i \nu_{\ell}\right) \\
& \times G_{\sigma}^{\mathrm{H}}\left(p-q, i \omega_{n}-i \nu_{\ell}\right),
\end{aligned}
$$

which is topologically equivalent to the first diagram in Fig. 1(d) with replacing $G^{0}$ with $G^{\mathrm{H}}$. If $\chi(q, 0)$ has a divergent behavior associated with the 1D nesting, one can also obtain the approximate self-energy inducing the Peierls pseu$\operatorname{dogap} \Delta_{\text {Pi. }}[41,101]$ as $\Sigma_{\sigma}^{2 \text { nd }}\left(p, i \omega_{n}\right) \simeq \Delta_{\text {Pi. }}^{2} G_{\sigma}^{\mathrm{H}}\left(p \pm 2 k_{\mu}, i \omega_{n}\right)$. Within the framework of FLEX and parquet approximations, one may expect the competition of two pseudogaps originating from Cooper and Peierls instabilities, even in this simple model at higher density or lower temperature, which is left as interesting future work.

Finally, in Fig. 5, we have also plotted the polarization $P=\left(n_{\uparrow}-n_{\downarrow}\right) /\left(n_{\uparrow}+n_{\downarrow}\right)$ in the presence of finite fictitious magnetic-field $h / T$. We compare our results with the CL method [83] which is developed to avoid a possible sign problem in polarized systems. We note that the CL method also agrees with other methods, such as lattice simulation with an analytic continuation from the imaginary chemical potential $[67,70,83]$. Our results well reproduce the CL results, indicating that our approach enables us to evaluate fluctuation effects quantitatively even in the presence of polarization. Thus, one can expect possible future applications of our diagrammatic approach to other interesting problems, such as fluctuation effects on FFLO-like pairing states, transport in quantum wires [102], and multipolaronic excitations $[103,104]$.

\section{SUMMARY}

To summarize, we have investigated low-dimensional fluctuation effects in an attractive Gaudin-Yang Fermi gas at finite temperature within the diagrammatic approach. We have developed the many-body $T$-matrix theory combined with the self-consistent Hartree approximation to incorporate pairing fluctuation effects and a nonvanishing mean-field shift, respectively.

The calculated number densities and polarizations in unpolarized and polarized gases show excellent agreement with the recent QMC and CL results in the wide ranges of an interaction parameter and chemical potentials. These results indicate the reliability of our approach in the region where we have explored in this paper. The single-particle spectral functions exhibit the crossover from the superposition of bare and dressed atomic states to the pseudogap state with increasing the interaction strength and the number density. These excitation properties can partially be captured by the extraction of the dispersion from the imaginary-time Green's function. Our results would contribute to further understanding of strongcoupling nature in low-dimensional systems.

In this paper, whereas we consider a uniform 1D system, it is worth studying the dimensional crossover as well as mixeddimensional systems in order to find relations to pseudogap phenomena in higher-dimensional systems. Furthermore, our approach can be extended to the mass-imbalanced mixtures and the trapped systems. It is also interesting to address photoemission spectra which can be experimentally measured.

\section{ACKNOWLEDGMENTS}

The authors thank J. E. Drut and A. C. Loheac for sharing their numerical data with us, $\mathrm{K}$. Iida for reading the paper and giving insightful comments, and T. Hatsuda, M. Horikoshi, S. Inoue, K. Kato, and Y. Sekino for useful discussions. This work was supported by Grants-in-Aid for JSPS fellows (Grant No. 17J03975) and for Scientific Research from JSPS (Grants No. $18 \mathrm{H} 05406$ and No. 20K14480). S.T. was supported by the RIKEN Special Postdoctoral Researchers Program.
[1] T. Timusk and B. Statt, Rep. Prog. Phys. 62, 61 (1999).

[2] Y. Yanase, T. Jujo, T. Nomura, H. Ikeda, T. Hotta, and K. Yamada, Phys. Rep. 387, 1 (2003).

[3] Ø. Fischer, M. Kugler, I. Maggio-Aprile, C. Berthod, and C. Renner, Rev. Mod. Phys. 79, 353 (2007).

[4] C. Varma, Nature (London) 468, 184 (2010).
[5] M. Hashimoto, I. M. Vishik, H. He, T. P. Devereaux, and Z.-X. Shen, Nat. Phys. 10, 483 (2014).

[6] Q. Chen, J. Stajic, S. Tan, and K. Levin, Phys. Rep. 412, 1 (2005).

[7] I. Bloch, J. Dalibard, and W. Zwerger, Rev. Mod. Phys. 80, 885 (2008). 
[8] S. Giorgini, L. P. Pitaevskii, and S. Stringari, Rev. Mod. Phys. 80, 1215 (2008).

[9] The BCS-BEC Crossover and the Unitary Fermi Gas, edited by W. Zwerger, Lecture Notes in Physics Vol. 836 (Springer, Berlin, 2012).

[10] M. Randeria and E. Taylor, Annu. Rev. Condens. Matter Phys. 5, 209 (2014).

[11] E. J. Mueller, Rep. Prog. Phys. 80, 104401 (2017).

[12] G. C. Strinati, P. Pieri, G. Röpke, P, Schuck, and M. Urban, Phys. Rep. 738, 3 (2018).

[13] S. Jensen, C. N. Gilbreth, and Y. Alhassid, Eur. Phys. J.: Spec. Top. 227, 2241 (2019).

[14] Y. Ohashi, H. Tajima, and P. van Wyk, Prog. Part. Nucl. Phys. 111, 103739 (2020).

[15] A. Schnell, G. Röpke, and P. Schuck, Phys. Rev. Lett. 83, 1926 (1999).

[16] M. Kitazawa, T. Koide, T. Kunihiro, and Y. Nemoto, Phys. Rev. D 65, 091504(R) (2002).

[17] M. Kitazawa, T. Kunihiro, and Y. Nemoto, Phys. Lett. B 633, 269 (2006).

[18] T. Abe and R. Seki, Phys. Rev. C 79, 054002 (2009).

[19] X.-G. Huang, Phys. Rev. C 81, 034007 (2010).

[20] J. Pang, J. Wang, and L. He, Phys. Rev. D 88, 054017 (2013).

[21] J. T. Stewart, J. P. Gaebler, and D. S. Jin, Nature (London) 454, 744 (2008).

[22] R. Haussmann, M. Punk, and W. Zwerger, Phys. Rev. A 80, 063612 (2009).

[23] Q. Chen and K. Levin, Phys. Rev. Lett. 102, 190402 (2009).

[24] S. Tsuchiya, R. Watanabe, and Y. Ohashi, Phys. Rev. A 80, 033613 (2009).

[25] S.-Q. Su, D. E. Sheehy, J. Moreno, and M. Jarrell, Phys. Rev. A 81, 051604(R) (2010).

[26] H. Hu, X.-J. Liu, P. D. Drummond, and H. Dong, Phys. Rev. Lett. 104, 240407 (2010).

[27] J. P. Gaebler, J. T. Stewart, T. E. Drake, D. S. Jin, A. Perali, P. Pieri, and G. C. Strinati, Nat. Phys. 6, 569 (2010).

[28] A. Perali, F. Palestini, P. Pieri, G. C. Strinati, J. T. Stewart, J. P. Gaebler, T. E. Drake, and D. S. Jin, Phys. Rev. Lett. 106, 060402 (2011).

[29] P. Magierski, G. Wlazłowski, and A. Blugac, Phys. Rev. Lett. 107, 145304 (2011).

[30] G. Wlazłowski, P. Magierski, J. E. Drut, A. Bulgac, and K. J. Roche, Phys. Rev. Lett. 110, 090401 (2013).

[31] Y. Sagi, T. E. Drake, R. Paudel, R. Chapurin, and D. S. Jin,, Phys. Rev. Lett. 114, 075301 (2015).

[32] S. Jensen, C. N. Gilbreth, and Y. Alhassid, Phys. Rev. Lett. 124, 090604 (2020).

[33] A. Richie-Halford, J. E. Drut, and A. Bulgac, Phys. Rev. Lett. 125, 060403 (2020).

[34] D. M. Eagles, Phys. Rev. 186, 456 (1969).

[35] A. J. Leggett, in Modern Trends in the Theory of Condensed Matter, edited by A. Pekalski and J. Przystawa (Springer Verlag, Berlin, 1980), p. 14.

[36] P. Nozières and S. Schmitt-Rink, J. Low Temp. Phys. 59, 195 (1985).

[37] C. A. R. Sa de Melo, M. Randeria, and J. R. Engelbrecht, Phys. Rev. Lett. 71, 3202 (1993).

[38] Y. Ohashi and A. Griffin, Phys. Rev. Lett. 89, 130402 (2002).

[39] C. A. Regal, M. Greiner, and D. S. Jin, Phys. Rev. Lett. 92, 040403 (2004).
[40] M. W. Zwierlein, C. A. Stan, C. H. Schunck, S. M. F. Raupach, A. J. Kerman, and W. Ketterle, Phys. Rev. Lett. 92, 120403 (2004).

[41] P. A. Lee, T. M. Rice, and P. W. Anderson, Phys. Rev. Lett. 31, 462 (1973).

[42] A. Luther and I. Peschel, Phys. Rev. B 9, 2911 (1974).

[43] M. S. Dresselhaus, G. Dresselhaus, and R. Saito, Carbon 33, 883 (1995).

[44] V. Meunier, A. G. Souza Filho, E. B. Barros, and M. S. Dresselhaus, Rev. Mod. Phys. 88, 025005 (2016).

[45] W. A. Little, Phys. Rev. 134, A1416 (1964).

[46] A. J. Heeger, S. Kivelson, J. R. Schrieffer, and W.-P. Su, Rev. Mod. Phys. 60, 781 (1988).

[47] D. Jérome, Chem. Phys. 104, 5565 (2004).

[48] G. Watanabe, K. Iida, and K. Sato, Nucl. Phys. A 676, 455 (2000).

[49] M. E. Caplan and C. J. Horowitz, Rev. Mod. Phys. 89, 041002 (2017).

[50] M. Feld, B. Fröhlich, E. Vogt, M. Koschorreck, and M. Köhl, Nature (London) 480, 75 (2011).

[51] B. Fröhlich, M. Feld, E. Vogt, M. Koschorreck, W. Zwerger, and M. Köhl, Phys. Rev. Lett. 106, 105301 (2011).

[52] V. Pietilä, Phys. Rev. A 86, 023608 (2012).

[53] S. N. Klimin, J. Tempere, and J. T. Devreese, New J. Phys. 14, 103044 (2012).

[54] V. Ngampruetikorn, J. Levinsen, and M. M. Parish, Phys. Rev. Lett. 111, 265301 (2013).

[55] R. Watanabe, S. Tsuchiya, and Y. Ohashi, Phys. Rev. A 88, 013637 (2013).

[56] M. Bauer, M. M. Parish, and T. Enss, Phys. Rev. Lett. 112, 135302 (2014).

[57] F. Marsiglio, P. Pieri, A. Perali, F. Palestini, and G. C. Strinati, Phys. Rev. B 91, 054509 (2015).

[58] P. A. Murthy, M. Neidig, R. Klemt, L. Bayha, I. Boettcher, T. Enss, M. Holten, G. Zürn, P. M. Preiss, and S. Jochim, Science 359, 6374 (2018).

[59] M. Matsumoto, R. Hanai, D. Inotani, and Y. Ohashi, J. Phys. Soc. Jpn. 87, 014301 (2018).

[60] B. C. Mulkerin, X.-J. Liu, and H. Hu, Phys. Rev. A 102, 013313 (2020).

[61] X.-W. Guan, M. T. Batchelor, and C. Lee, Rev. Mod. Phys. 85, 1633 (2013).

[62] A. Imambekov, T. L. Schmidt, and L. I. Glazman, Rev Mod. Phys. 84, 1253 (2012).

[63] M. Takahashi, Thermodynamics of One-Dimensional Solvable Models (Cambridge University Press, Cambridge, U.K., 1999).

[64] X.-W. Guan, M. T. Batchelor, C. Lee, and M. Bortz, Phys. Rev. B 76, 085120 (2007).

[65] X.-W. Guan, X, -G. Yin, A. Foerster, M. T. Batchelor, C.-H. Lee, and H.-Q. Lin, Phys. Rev. Lett. 111, 130401 (2013).

[66] M. D. Hoffman, P. D. Javernick, A. C. Loheac, W. J. Porter, E. R. Anderson, and J. E. Drut, Phys. Rev. A 91, 033618 (2015).

[67] A. C. Loheac, J. Braun, J. E. Drut, and D. Roscher, Phys. Rev. A 92, 063609 (2015).

[68] L. Rammelmüller, W. J. Porter, J. E. Drut, and J. Braun, Phys. Rev. D 96, 094506 (2017).

[69] C. R. Shill and J. E. Drut, Phys. Rev. A 98, 053615 (2018). 
[70] A. Alexandru, P. F. Bedaque, and N. C. Warrington, Phys. Rev. D 98, 054514 (2018).

[71] P. Fulde and R. A. Ferrell, Phys. Rev. 135, A550 (1964).

[72] A. Larkin and Y. Ovchinnikov, Zh. Eksp. Teor. Fiz. 47, 1136 (1964) [Sov. Phys. JETP 20, 762 (1965)].

[73] H. Hu, X.-J. Liu, and P. D. Drummond, Phys. Rev. Lett. 98, 070403 (2007).

[74] M. M. Parish, S. K. Baur, E. J. Mueller, and D. A. Huse, Phys. Rev. Lett. 99, 250403 (2007).

[75] G. Orso, Phys. Rev. Lett. 98, 070402 (2007).

[76] E. Zhao and W. V. Liu, Phys. Rev. A 78, 063605 (2008).

[77] M. R. Bakhtiari, M. J. Leskinen, and P. Törmä, Phys. Rev. Lett. 101, 120404 (2008).

[78] Y.-A. Liao, A. S. C. Rittner, T. Paprotta, W. Li, G. B. Partridge, R. G. Hulet, S. K. Baur, and E. J. Mueller, Nature (London) 467, 567 (2010).

[79] L. Rammelmüller, J. E. Drut, and J. Braun, SciPost Phys. 9, 014 (2020).

[80] E. Nakano and T. Tatsumi, Phys. Rev. D 71, 114006 (2005).

[81] K. Fukushima and T. Hatsuda, Rep. Prog. Phys. 74, 014001 (2011).

[82] M. Buballa and S. Carignano, Prog. Part. Nucl. Phys. 81, 39 (2015).

[83] A. C. Loheac, J. Braun, and J. E. Drut, Phys. Rev. D 98, 054507 (2018).

[84] C. E. Berger, L. Rammelmüller, A. C. Loheac, F. Ehmann, J. Braun, and J. E. Drut, arXiv:1907.10183.

[85] F. Werner and Y. Castin, Phys. Rev. A 86, 013626 (2012).

[86] M. Jin, M. Urban, and P. Schuck, Phys. Rev. C 82, 024911 (2010).

[87] S. Ramanan and M. Urban, Phys. Rev. C 88, 054315 (2013).
[88] P. van Wyk, H. Tajima, D. Inotani, A. Ohnishi, and Y. Ohashi, Phys. Rev. A 97, 013601 (2018).

[89] H. Tajima, T. Hatsuda, P. van Wyk, and Y. Ohashi, Sci. Rep. 9, 18477 (2019).

[90] E. V. H. Doggen and J. J. Kinnunen, Phys. Rev. Lett. 111, 025302 (2013).

[91] W.-B. He, Y.-Y. Chen, S. Zhang, and X.-W. Guan, Phys. Rev. A 94, 031604(R) (2016).

[92] J. Voit, Eur. Phys. J. B. 5, 505 (1998).

[93] E. Orignac and D. Poilblanc, Phys. Rev. B 68, 052504 (2003).

[94] J. N. Fuchs, A. Recati, and W. Zwerger, Phys. Rev. Lett. 93, 090408 (2004).

[95] H. J. Rothe, Lattice Gauge Theories (World Scientific, Singapore, 2012), and its references.

[96] N. D. Mermin and H. Wagner, Phys. Rev. Lett. 17, 1133 (1966).

[97] P. Hohenberg, Phys. Rev. 158, 383 (1967).

[98] I. E. Dzyaloshinskii and A. I. Larkin, Zh. Eksp. Teor. Fiz. 65, 411 (1973) [Sov. Phys. JETP 38, 202 (1974)].

[99] S. X. Yang, H. Fotso, J. Liu, T. A. Maier, K. Tomko, E. F. D'Azevedo, R. T. Scalettar, T. Pruschke, and M. Jarrell, Phys. Rev. E 80, 046706 (2009).

[100] A. L. Fetter and J. D. Walecka, Quantum Theory of ManyParticle Systems (Dover, Mineola, NY, 2003).

[101] L. Bartosch, Ann. Phys. (NY) 10, 799 (2001).

[102] A.-M. Visuri, M. Lebrat, S. Häusler, L. Corman, and T. Giamarchi, Phys. Rev. Res. 2, 023062 (2020).

[103] A. S. Dehkharghani, A. G. Volosniev, and N. T. Zinner, Phys. Rev. Lett. 121, 080405 (2018).

[104] S. I. Mistakidis, G. C. Katsimiga, G. M. Koutentakis, and P. Schmelcher, New J. Phys. 21, 043032 (2019). 